\title{
Modelling for Anaemia and Malnutrition in Children Less Than Five Years of Age in Lesotho: A Case Study of a Cross-Sectional Dataset Using Multivariate Joint Model.
}

\section{Rugiranka Tony Gaston ( $\nabla$ rugiratony@gmail.com )}

University of KwaZulu-Natal

\section{Shaun Ramroop}

University of KwaZulu-Natal

Faustin Habyarimana

University of KwaZulu-Natal

\section{Research Article}

Keywords: Malnutrition, anaemia, correlation, multivariate joint model, children less than five years of age and LDHS.

Posted Date: April 30th, 2021

DOl: https://doi.org/10.21203/rs.3.rs-403762/v1

License: (c) (1) This work is licensed under a Creative Commons Attribution 4.0 International License.

Read Full License 


\title{
Modelling for Anaemia and Malnutrition in Children Less than Five Years of Age in Lesotho: A Case Study of a Cross-Sectional Dataset Using Multivariate Joint Model.
}

\section{Rugiranka Tony Gaston ${ }^{1 *}$, Faustin Habyarimana ${ }^{2}$ and Shaun Ramroop ${ }^{2}$}

1. School of Mathematics, Statistics and Computer Sciences, University of KwaZuluNatal, Pietermaritzburg Campus, Private Bag X01, Scottsville, 3209, South Africa, and Health Economics and HIV/AIDS Research Division (HEARD), University of KwaZulu-Natal, Westville Campus, Private Bag X01, Westville, 3629, South Africa.

2. School of Mathematics, Statistics and Computer Sciences, University of KwaZuluNatal, Pietermaritzburg Campus, Private Bag X01, Scottsville, 3209, South Africa Correspondence: *rugiratony@gmail.com Tel: +27-033-260-5612.

\begin{abstract}
Background: Anaemia and malnutrition remain jointly a serious health issue worldwide especially in developing countries. In Lesotho, the prevalence of anaemia and malnutrition remains highly significant especially among children less than five years of age.
\end{abstract}

Objectives: The primary objective of the present study was to determine the association between anaemia and malnutrition, and identify the associated factors with the two diseases among children less than five years of age in Lesotho.

Methods: The present study was cross-sectional and used secondary data from the 2014 Lesotho Demographic Health Survey (LDHS). The study considered a total number of 3112 children and utilized a multivariate joint model under the scope of the generalized linear mixed model (GLMM) for the data analysis.

Results: The prevalence of anaemia and stunting in children less than five years of age were $51 \%$ and $43 \%$ respectively. The multivariate results revealed that there is a strong relationship between anaemia and malnutrition. In addition, the results indicated that education, residence, wealth index and childbirth weight have a significant effect on stunted or malnourished child, 
while child with fever and diarrhoea have a significant effect on anaemia. Lastly, child's age has a significant effect on both stunting and anaemia.

Conclusion: The study indicated a strong association between anaemia and stunting or malnutrition in the long-term, and controlling one disease should result in reducing the other. The socio-economic, geographical and demographic variables have a significant impact on improving stunting and anaemia. Thus, improving nutrition in children less than five years, especially those from rural area and having a birthweight less than $2500 \mathrm{~g}$, and educating the mothers to take their children to the health care facility when they have fever and diarrhoea, especially those from poor background, would contribute immensely to the reduction of malnutrition and anaemia in children from Lesotho.

Keywords: Malnutrition, anaemia, correlation, multivariate joint model, children less than five years of age and LDHS.

\section{Introduction}

Malnutrition and anaemia jointly remain health problems worldwide especially in developing countries (Leal et al., 2011; Yang et al., 2012; Aheto et al., 2015). Malnutrition and anaemia are the main causes of morbidity and mortality worldwide, particularly in children and pregnant women (Black et al., 2013; Kavosi et al., 2014; Aheto et al., 2017).

Anaemia is defined as a low level of haemoglobin concentration $(\mathrm{Hb})$ in the blood. According to the World Health Organization (WHO), children less than five years of age are considered anaemic when the $\mathrm{Hb}$ level adjusted for altitude is less than $11 \mathrm{~g} / \mathrm{dl}$ (Gayawan et al., 2014; WHO, 2015; Kejo et al., 2018). The cause of anaemia is multifactorial and iron deficiency contributes about $50 \%$ of the total causes. Insufficient folate, vitamin B12, protein deficiencies, nutrients and some parasitic diseases, such as malaria and diarrhoea among others, can also contribute in increasing anaemia (El Kishawi et al., 2015; Gaston et al., 2018). Childhood anaemia can have a negative effect on cognitive development, performance in school, physical growth and immunity (Sachdev et al., 2005; Kounnavong et al., 2011; Gaston et al., 2018).

Malnutrition is a condition that appears as insufficient food intake and other mineral resources or consuming too much nutrient. In developing countries, the nutritional status of a child can be used as an indicator of health problems (Masibo and Makoka, 2012). Childhood malnutrition 
can also result in negative effects in the long run, such as poor performance at school, poor psychomotor development, lower capacity for work and lower quality of life in adulthood (Okoromah et al., 2011; Black et al., 2013; Kassebaum et al., 2017).

The nutritional status of a child is mainly evaluated using the anthropometry method, and this method uses different indicators to decide whether an individual is malnourished or nourished (normal nutritional status) (MOHSW and ICF, 2016). Stunting indicator (short height-for-age) indicates chronic or long-term malnutrition, wasting (low-weight-for-height) indicates a failure to receive adequate food intake or a recent period of illness causing the loss of weight (acute malnutrition), while underweight is the composite index of wasting and stunting (MOHSW and ICF, 2016).

The three anthropometric variables are measured based on Z-score and the higher value of Zscore, the better the nutrition status. The WHO describes an individual as stunting, wasting or underweight when their z-score falls below minus two standard deviations (SD) (Bardosono et al., 2007; Kazembe et al., 2013; Anticona and Sebastian, 2014; MOHSW and ICF, 2016). Stunting, wasting and underweight can be used independently or together depending on the reason for the assessment and the idea of mediation. For instance, when the goal is to get prompt ideas of a community or a large number of people to understand the extent of information in the short term, the wasting measurement would be useful to provide adequate information. When the target to assess the nutritional status of an individual's group is for the long term, stunting can be helpful. However, when the target is to get information and decide the kind of programs to implement in a specific region, the study can include all three anthropometric measurements (Setboonsarng, 2005; Rahman et al., 2019). The present study used stunting as an indicator of long-term malnutrition to examine the correlation between anaemia and malnutrition (Olney et al., 2009; Mohammed et al., 2019; Rahman et al., 2019).

Despite the effort and progress made to improve the childhood nutrition status and fight against anaemia, nonetheless, challenges remain. Globally in 2017, 151 million children less than five years of age were stunted, 51 million wasted and 38 million overweight. Among those children, three quarters were from the South-East Asian and African regions (UNICEF, 2017). The prevalence of anaemia in children less than five years in 2011 was approximately $43 \%$ globally, and the highest number was from the African and South Asian regions (Milman, 2011; WHO, 2011; Kejo et al., 2018). 
In Lesotho, anaemia and malnutrition in children less than five years remains a health problem, where 51\% and 53\% are anaemic and malnourished, respectively (MOHSW and ICF, 2016). These percentages indicate that the prevalence of the two diseases are still high in that country. Therefore, an improvement in health care, nutrition and reducing anaemia in children less than five years of age is most important as they are the future leaders of the country (Gaston et al., 2018). In addition, understanding the link between anaemia and malnutrition and other associated factors will help the policy-makers and donors in planning and focussing on children less than five years of age. In Lesotho, studies about anaemia and malnutrition in children less than five years are limited, to name a few such as Worku et al. (2003); Renzaho (2006); Gaston et al. (2018); and Letuka and Frade (2020). All the above-mentioned studies were interested in modelling anaemia or malnutrition and other associated factors separately, and those models have their own advantages; however, they cannot address the association between the two diseases.

Generally, previous studies interested in modelling anaemia and malnutrition simultaneously, such as those by Olney et al. (2009), Yang et al. (2012), Adeyemi et al. (2019), and Rivadeneira et al. (2020) among others, showed a significant effect between the two diseases.

However, the prevalence of anaemia or malnutrition differs in each country and can depend on variability of accessing health care and the food intake with enough nutrients across different countries (Gaston et al., 2018).

Based on literature and our understanding, no study has been carried out in Lesotho using a multivariate joint model for anaemia and malnutrition simultaneously among children less than five years of age. The present study used multivariate joint model under the scope of Generalized Linear Mixed Model (GLMM) developed by Breslow and Clayton (1993) to include the random effect in the model. The random effect is unable to model the correlation between one or more responses variables (Hedeker, 2005; Agresti, 2015; Gaston and Ramroop, 2020). Furthermore, the multivariate under a GLMM has focal points in comparison of the separated model, such as controlling type 1 error rate in the various tests. In addition, the multivariate joint model is better for expansion in the capacity of the parameter estimate and the capacity to address particular questions (Guerguieva, 2001; Molenberghs and Verbeke, 2005). 
Therefore, the present study contributes to existing separated models for anaemia and malnutrition into multivariate joint model using generalized linear mixed model (GLMM) to simultaneously model the association between anaemia and malnutrition and explore other associated factors, which highlights the uniqueness of this study.

\section{Materials and Methods}

\subsection{Study area}

Lesotho is one of the smallest landlocked countries in Southern Africa and is surrounded only by South Africa. Lesotho has a total surface area of 30355 square kilometres with an estimated population of about of 2.2 million. Lesotho has ten politico-administrative districts with Maseru as the capital city, and is ruled by a King as the head of state and the Prime Minister rules the country as head of the government. The kingdom of Lesotho is known for its abundant water resources and high altitude. In addition, the kingdom of Lesotho has a high unemployment rate, HIV and AIDS problem, poverty, food insecurity, and other diseases, which increase the morbidity and mortality in the country (MOHSW and ICF, 2016). The country is also vulnerable to natural disasters and climate change such as droughts and heavy rain and flooding (Renzaho, 2015).

\subsection{Data source and sampling techniques}

The present study was a cross-sectional dataset and used secondary data from the Lesotho Demographic and Health Survey (LDHS), which was conducted in four months from September to December 2014. The ethical approval for the 2014 LDHS was assessed and confirmed by the Lesotho Ministry of Health Research and Ethics Committee together with support of the Institutional Review Board of ICF International. In addition, all children aged 6-59 months who were presented on the day of survey were tested for anaemia, and stunting under the supervision of their parents or guardians.

The 2014 survey followed a two-stage sampling design and was held at national level, in urban and rural areas, comprising four ecological zones and each district in Lesotho. The two-stage sampling design included the cluster sampling of enumeration areas and systematic sampling of households. The first stage included selecting clusters, consisting of enumeration areas, and a total number of 40 clusters were selected, where 118 and 282 were from urban and rural areas, respectively. The second stage involved systematic sampling of 9942 households, and 
households included in the survey were randomly selected in all enumeration areas (EAs). Out of the total number of 9942 households, 25 households were selected from each enumeration area (MOHSW and ICF, 2016).

Only children aged 0-59 months from the mothers who stayed in or visited the household the night before the 2014 survey were included. The present study used the children's file record and a total weighted number of 3112 children were drawn as a sample size to demonstrate a national-level representation (MOHSW and ICF, 2016; Gaston and Ramroop, 2020). The weighted samples were used to achieve the standard that is descriptive of the nation and accounts for the complex sample design from the data. The population in each region did have not the same weight and the individuals surveyed in each region should contribute equivalently to the size of the total number in each region. To overcome this problem of unweighted, the present study used a weighted sample size (MOHSW and ICF, 2016; Gaston and Ramroop, 2020).

\subsection{Blood collection for anaemia and nutrition assessment for the children}

Anaemia testing: All children aged 6-59 months who were presented on the day of survey were tested for anaemia under the supervision of their parents or guardians. The professional nurses did the testing and the collection of blood samples was done by using a spring-loaded, sterile lancet to make a finger- or heel-prick. The lancet, microcuvette, gloves and alcohol swabs were used once for hygiene safety. The drop of blood was gathered in a microcuvette, and the Haemoglobin level was examined using a convenient HemoCue analyser.

The outcomes were accessible within 10 minutes and were given to the children's parents or guardians verbally and recorded as a hard copy. All children with a haemoglobin level less than $8 \mathrm{~g} / \mathrm{dl}$ were considered as anaemic and were given medicine on the spot as per the public health standards (MOHSW and ICF, 2016). In addition, the parents or guardians were advised to take their children to the nearest healthcare facility for follow-up, and were given a referral letter to show the staff at the health care facility. The anaemia test results were captured on the Biomarker Questionnaire and on the handout that contains data on the causes and counteraction of anaemia and were left in the family unit (MOHSW and ICF, 2016).

Nutrition status assessment: The anthropometric parameters included measurement of height with a tape board and weight with an electronic balance provided by UNICEF. The weight of 
the children was estimated utilizing a Seca gauging scale, which was aligned to zero. Parents or guardians were asked to take off the clothes of their children or dress them in light clothes, and stand them on the weighing scale till their weight was recorded. For children who were not able to stand, first the weight of their parents was taken and then the weight of parents holding their children. In order to get the weight for the children, the weight of the parents was deducted from the combined weight of both parents and children. The height measurement was carried out using a short board which was lying down or standing on a level ground surface. The parents were asked to remove the children's shoes and headgear; the children were helped to stand against the board against the wall. However, the children who were less than two years or less than $87 \mathrm{~cm}$ were measured lying down on the board. Thereafter, the nutrition status of the children was determined using weight, height and age of the children (Bechir et al., 2012; El Kishawi et al., 2015; MOHSW and ICF, 2016).

\section{Data Analysis}

\subsection{Dependent variable}

The response variables from the present study were dichotomous, and the first dichotomous outcome variable was the child is either anaemic or not. Based on the WHO guidelines the child was recorded as anaemic, when the haemoglobin concentration level adjusted for altitude was less than $11 \mathrm{~g} / \mathrm{dl}$ (WHO, 2015). The second response variable was stunting, which is an indicator of malnutrition in the long term, and was used to check whether a child was stunted or not stunted (Rahman et al., 2019). The WHO describes an individual as stunting when the z-score falls below minus two standard deviations (Kazembe et al., 2013; MOHSW and ICF, 2016)

\subsection{Independent variables}

The independent variables viewed in this study included a number of socio-economic and demographic factors. These variables were also included in previous studies on childhood anaemia or malnutrition such as the study by Gaston et al. (2018). These variables comprised the age of the child in months; sex of the child; mother's education level; type of place of residence; whether or not household had electricity; main material of the floor; main material of the wall; main material of the roof; wealth index; child's birth weight; had fever in last two weeks; had diarrhoea recently; had cough in last two weeks; received vitamin A in last 6 months; birth order; mother's BMI; mother's education level; visited health facility; toilet 
facility; and type of drinking water (Gaston et al., 2018; Gaston and Ramroop, 2020; Letuka and Frade, 2020).

\section{Statistical Analysis}

The present study used univariate techniques to indicate the association between the independent and dependent (responses) variables. The multivariate technique was used to identify the correlation between two response variables (anaemia and malnutrition) and the associated factors. The cleaning and analysis of univariate data was done in Statistical Package for the Social Sciences (SPSS). The univariate analysis used cross tabulation techniques to summarise the data using frequencies and percentages, and investigate the relationship between the responses variables and independent variables using p-values. The independent variables with p-value less than 0.2 were included in the multivariate generalized linear mixed model (GLMM). The process of including all independent variables less than 0.2 was due to account for multicollinearity and confounding, which might be between the covariates (El Kishawi et al., 2015; Gari et al., 2017, Gaston et al., 2018). The multivariate analysis reported only the independent variable found to be statistically significant with response variables at $p$-value less than five percent (0.05). SAS 9.4 was used in analysis of multivariate under GLMM using PROC GLIMMIX procedure. This procedure is good in joining models with two response variables, which have a similar distribution and link function. Based on the convergence criteria the unstructured (UN) convergence seemed to be the best for the analysis. The present study checked the interaction effect based on the lowest Akaike information criteria (AIC); however, none was found to be statistically significant (Molenberghs and Verbeke, 2005; Gaston et al., 2020).

\subsection{Model formulation}

The present study examined the two responses variables, which were nutrition and the anaemia status of the children less than five years of age. Let us assume the first response variable $x_{i 1}$ to be nutrition status, where number one is assigned to a positive and zero to a negative child. The second response variable as $x_{i 2}$ to be anaemia status, where number one is allocated to an anaemic child and number zero to a non-anaemic child. Assume that the outcome is from a bivariate Bernoulli distribution, with $p_{i 1}$ as the likelihood of malnutrition occurring in a child $i$ and $p_{i 2}$ as the probability of anaemia occurring in a child $i$. Therefore, the binary generalized linear model can be expressed as follow: 
$f_{1}\left(\psi_{i 1}\right)=Y_{i 1} \theta_{1}+Z_{i 1} u_{1}$

$f_{2}\left(\psi_{i 1}\right)=Y_{i 2} \theta_{2}+Z_{i 2} u_{2}$.

Where, $\theta_{1}$ and $\theta_{2}$ are assumed to be the vectors of fixed effects, $u_{1}$ and $u_{2}$ are the vectors of the random effects. In addition, $Y_{i 1}, Y_{i 2}, Z_{i 1}$ and $Z_{i 2}$ are the designed matrices for fixed effects and random effects respectively. Hence, the equation of the variance-covariance matrices model is shown as follows:

$U=\left(\begin{array}{l}u_{1} \\ u_{2}\end{array}\right) \sim$ i.i.d.MVN $(0, \sigma)=\operatorname{MVN}\left(\left[\begin{array}{l}0 \\ 0\end{array}\right],\left[\begin{array}{ll}\sigma_{11} & \sigma_{12} \\ \sigma_{21} & \sigma_{22}\end{array}\right]\right)$

Where the $\sigma_{11}$ and $\sigma_{22}$, are the variance components of stunting of children under five years and anaemia respectively, while $\sigma_{12}$ and $\sigma_{21}$ are the correlation components between stunting and anaemia. When the correlation components from equation (3), $\sigma_{12}=\sigma_{21}=0$, the multivariate joint model under generalized linear mixed model becomes a separate model (Molenberghs and Verbeke, 2005; Habyarimana et al., 2016).

\subsection{Model testing and and goodness-of-fit}

Covariance structure of the random effects: The test of random effect is necessary to check whether the random effect for a specific covariate should be included in the model or not and compares to testing the hypothesis that the variance of population distribution is zero. Assume that we want to test a hypothesis about the variance parameter $\sigma^{2}$ for GLMM with a single random effect. Since the variance cannot be negative, zero will be at the boundary of the parameter space, and one tests a one-sided hypothesis (Verbeke and Molenberghs, 2003; Tuerlinckx et al., 2006).

The null hypothesis to be tested is $H_{0}: \sigma^{2}=0$, against $H_{1}: \sigma^{2} \neq 0$. This hypothesis test can be tested using the likelihood ratio test, the Wald test or the score test. Such tests are asymptotically comparable under the null hypothesis and follow a distribution with a given degree of freedom. In addition, these tests can be used to test if the random effects in a GLMM is significant or not (Verbeke and Molenberghs, 2003; Tuerlinckx et al., 2006).

Goodness-of-fit tests: The goodness-of-fit can be evaluated by a likelihood ratio test, and its alternative model is saturated, which is the most complex model explained by the data. The saturated model can be expressed as $\boldsymbol{E}\left(\boldsymbol{Y}_{\boldsymbol{n} i} / \boldsymbol{\beta}, \boldsymbol{\theta}_{\boldsymbol{n}}\right)=\boldsymbol{Y}_{\boldsymbol{n} \boldsymbol{i}}$ for all $n$ and $i$. When the dependent 
variables are categorical, the goodness-of-fit test for the data is Pearson chi-squared test (Tuerlinckx et al., 2006). The Pearson chi-squared test can be written as follows:

$$
\chi^{2}=\sum_{i=1}^{I} n_{i} \sum_{j=0}^{J} \frac{\left(y_{i j}-\widehat{\pi}_{i j}\right)^{2}}{\widehat{\pi}_{i j}}
$$

Where $n_{i}$ is the number of individuals who participated with the same values on the covariates, $y_{i j}$ and $\hat{\pi}_{i j}$ are respectively the observed and expected quantity of the participants in the group $i$ responding in category $j$ (Tuerlinckx et al., 2006).

\section{Results and Interpretation}

\subsection{Univariate results}

The data analysis of this study comprised of a total weighted number of 3112 children aged between 0-59 months. The prevalence of anaemia and stunting in children less than five years of age was $51 \%$ and $43 \%$ respectively. Table 1 and Table 2 indicate the frequency distribution and percentage of anaemia with corresponding independent variables. Table 1 indicated that the child's age, children who had fever in last two weeks, had diarrhoea recently, and main roof material were statistically significant with childhood anaemia. Table 2 showed that the child's age, sex of a child, children who visited health care facilities, mother's education level, and wealth index, household with electricity, toilet facility, and type of drinking water, main wall and main floor material were statistically significant with childhood stunting.

Table 1 showed that the prevalence of anaemia was higher in the child age group 0-19 months (62.7\%), and decreased as the age increased by the age group 20-39 months (55.0\%), and age group 40-59 months (44.4\%) respectively. The prevalence of anaemia in children who had fever in last two weeks was higher $(62.8 \%)$ compared to children with no fever $(37.2 \%)$.

Table 2 indicated that the prevalence of stunted child was lower in age group 0-19 months, (17.8\%), and increased as the child grew older 20-39 months (33.3\%), and 40-59 months $(31.2 \%)$ respectively. 
Table 2 also indicated that the prevalence of stunting was higher in children from mothers with no education $(72.7 \%)$, and reduced as the level of mother's education increased by primary $(31.3 \%)$ and post-primary $(21.7 \%)$ respectively.

\section{Table 1: Childhood anaemia by categorical variable}

\section{Table 2: Childhood stunting by categorical variable}

\subsection{Multivariate results}

The findings from Table 4 indicate the variance components and covariance components between anaemia and stunting. The coefficient estimate of 1.000 revealed that there is a significant positive association between anaemia and stunting. This means that any change to either nutrition or anaemia status of a child can have a significant effect on each of the two diseases. In addition, the socio-economic demographic from Table 3 indicated a significant effect on both stunting and anaemia. Table 3 revealed that the child's age had a significant effect on both anaemia and stunting. Children aged less than 20 months were 0.436 times less likely to be stunted or malnourished compared to those in the reference group (40-59 months). However, children aged 20-39 months showed a significant effect on anaemia, and were 1.701 times more likely to have anaemia compared to those from the reference category.

The children with fever indicated a significant effect on anaemia, and the odds of having anaemia from children with no fever were 0.491 times less likely compared to children with fever. The same results show that children with no diarrhoea are 0.609 times less likely to have anaemia than those with diarrhoea. The childbirth weight has a significant effect on nutrition status of a child, and a child born with a higher weight $(\geq 2500 \mathrm{~g})$ is 0.240 times less likely to be stunted or malnourished than a child born with a lower weight $(<2500 \mathrm{~g})$. The same results also revealed that children living in rural areas were 0.520 times less likely to be stunted compared to those living in urban areas. Table 3 shows that the odds of being stunted for children from poorer classes were 3.501 times more likely to be stunted than those from richer classes. In addition, children from middle classes were 2.901 times more likely to be stunted compared to those from the reference category (Richer). Lastly, the same results indicated that 
children from mothers with post-primary education levels were 0.159 times less likely to be stunted compared to those from mothers with no education.

\section{Table 3. Parameter estimates for a joint marginal model for stunting and anaemia}

The findings from Table 4 indicate the variance components and covariance components between anaemia and stunting. The overall fitted model with the coefficient estimate of 1.000 and $p$-value $=0.000$ confirmed a positive significant association between anaemia and stunting. Therefore, the significant association between the two diseases confirmed that the random effect was necessary in the model (Molenberghs and Verbeke, 2005).

The test covariance parameters based on pseudo-likelihood rejected the null hypothesis of zero correlation with Pearson chi-squared test $=2644.470$ and $p$-value $=0.000$. This revealed that the correlation between anaemia and malnutrition was significant and not zero (Tuerlinckx et al., 2006).

Furthermore, the results from the fitted statistics for conditional distribution indicated the Pearson chi-squared $=2123.070$ with its degree of freedom $=0.94$. The degree of freedom closer to one means that there is a good variability in the dataset and there was no residual over dispersion (Molenberghs and Verbeke, 2005).

\section{Table 4. Variance Components}

\section{Discussion}

The present study was cross-sectional and used secondary data from 2014 LDHS. Based on the literature, this was the first study to model the association between anaemia and malnutrition simultaneously in children less than five years of age in Lesotho. The study utilized a multivariate joint model under the scope of generalized linear mixed model (GLMM) to simultaneously model the association between the two diseases and explore other associated factors. The present study revealed that there is a significant positive association between anaemia and stunting, and confirmed that the two diseases are still a health problem in children. 
This positive association means that the two diseases move in the same direction, and controlling malnutrition by improving the nutrition status of children could result in reducing anaemia. The same results were found in studies by Yang et al. (2012); Gari et al. (2017); Mohammed et al. (2019); Rahman et al. (2019) and Rivadeneira et al. (2020). The findings from this study indicate that the child's age has a significant effect on both anaemia and malnutrition. The chance of having anaemia or stunting (malnutrition) reduced as the children grew older (Anticona and Sebastian, 2014; Gari et al., 2017; Gaston et al., 2018; Kejo et al., 2018; Rahman et al., 2019). However, the studies by Anticona and Sebastian (2014) and Oliveira et al. (2015) confirmed that stunting increased as the children grew older.

The findings from this study revealed that fever has a significant effect on childhood anaemia and the odds of having anaemia was lower in children with no fever. Other authors have reported a significant association between fever and anaemia (Habyarimana et al., 2016; Gaston et al., 2018).

The same results showed that diarrhoea has a significant effect on anaemia, and the risk of having anaemia is high in children with diarrhoea. The findings from this study are in line with previous research (Gaston et al., 2018; Rivadeneira et al., 2020).

The findings from this study revealed that the probability of being malnourished reduced as the education levels of the mothers increased. The findings from this study are in line with previous studies such as Kavosi et al. (2014); Aheto et al. (2015); Adebayo et al. (2016); and Aheto et al. (2017).

The childbirth weight has a significant effect on nutrition status of children, and the risk of being malnourished is lower in children born with a higher weight $(\geq 2500 \mathrm{~g})$. Similar findings were found in studies by Yang et al. (2012); Habyarimana et al. (2016); and Kejo et. (2018).

The same results also revealed that children living in rural areas have a lower risk of having malnutrition. Similar results are shown in previous studies, such as the study by Kavosi et al. (2014). However, the results from the present study are in contrast with the study by Yang et al. (2012) and El Kishawi et al. (2015).

Lastly, the findings from this study indicated that children from middle and richer classes have a lower risk of having malnutrition (Gari et al., 2017; Rivadeneira et al., 2020). 


\section{Conclusion}

The primary objective of the present study was to determine the association between anaemia and malnutrition in children less than five years of age in Lesotho using multivariate joint model under generalized linear mixed model (GLMM).

The study also checked other factors which might be associated with anaemia and malnutrition. Lastly, we checked for any possible interaction effect between the independent variables and none was statistically significant, and therefore these were not included in the results. The study used stunting variable as an indicator of malnutrition in the long-term (Rahman et al., 2019). The findings revealed that there is a significant positive association between anaemia and malnutrition. This indicates that when malnutrition increases in children less than five years anaemia also increases and vice-versa. Thus, a change in nutrition status of a child can have a significant impact on anaemia status. In addition, the socio-economic and demographic indicated a significant impact on both malnutrition and anaemia.

The findings show that there is a need to educate mothers, especially those with no education, from rural area and with poorer background, on how to prevent anaemia by improving nutrition of their children. In addition, children with fever, birthweight less than $2500 \mathrm{~g}$, and have diarrhoea should be prioritized by improving their nutrition status and taken to the nearest healthcare facility for a check-up.

\section{Strengths and Limitation of the Study}

The present study used joint model to assess the association between anaemia and nutrition status in children less than five years of age. Knowledge on the relationship between anaemia and malnutrition together with other factors associated with the two conditions can provide useful insights to policy makers, donors and government in planning and fighting against malnutrition and anaemia. Furthermore, the statistical model used in this study can be useful to other researchers in referencing. However, the present study has some limitation, as the data set was cross-sectional and could not check the change in factors and prevalence of anaemia and malnutrition over time. To overcome that, future research could make use of longitudinal data set, and also use spatial joint model to assess the association between malnutrition and anaemia by geographical location. 


\section{Recommendations}

In order to fight against anaemia and malnutrition in children less than five years of age in Lesotho, policy makers, donors and government should focus on improving nutrition status especially in children from rural area, with diarrhoea, fever, birthweight less than $2500 \mathrm{~g}$, from poorer quantile index household and uneducated mother.

\section{Abbreviations}

OR: Odds ratio; SE: Standard error; LDHS: Lesotho demographic health survey; GLMM: Generalized linear mixed model; AIC: Akaike information criterion; Hb: haemoglobin concentration; UN: unstructured; WHO: World Health Organization

\section{Availability of data and materials}

The present study utilized existing dataset and is available from https://www.dhsprogram.com/data/dataset_admin/login_main.cfm with the permission from the DHS program.

\section{Ethics approval and consent to participate}

The ethical approval for the 2014 LDHS was approved by the Lesotho Ministry of Health Research and Ethics Committee together with support of the Institutional Review Board of ICF International. Verbal informed consent was obtained from children parents or guardians before conducting the surveys. The interviewers explained the procedure, the willingness to participate in the survey and the confidentiality of the data.

\section{Declarations}

\section{Author contribution statement}


R.T. Gaston: designed the study; acquired the data; performed the analysis; interpreted the results; and wrote the manuscript.

F. Habyarimana: Revised the manuscript and approved the final manuscript.

S. Ramroop: Revised the manuscript; and approved the final manuscript

\section{Funding statement}

This work was supported by the Health Economics and HIV/AIDS Research Division (HEARD).

\section{Competing interest statement}

The authors declare no conflict of interest.

\section{Consent for publication}

Not applicable.

\section{Additional information}

No additional information is available for this paper.

\section{Acknowledgements}

The authors acknowledge the Ministry of Health (Lesotho) and ICF International through the DHS programme for providing the data.

\section{References}

Adebayo, S.B., Gayawan, E., Heumann, C. and Seiler, C., 2016. Joint modeling of Anaemia and Malaria in children under five in Nigeria. Spatial and spatio-temporal epidemiology, 17, pp.105-115. 
Adeyemi, R.A., Zewotir, T. and Ramroop, S., 2019. Joint spatial mapping of childhood anaemia and malnutrition in sub-Saharan Africa: a cross-sectional study of small-scale geographical disparities. African Health Sciences, 19(3), pp.2692-2712.

Agresti, A., 2015. Foundations of linear and generalized linear models. John Wiley \& Sons, Inc. Hoboken, New Jersey.

Aheto, J.M.K., Keegan, T.J., Taylor, B.M. and Diggle, P.J., 2015. Childhood Malnutrition and Its Determinants among Under-Five Children in Ghana. Paediatric and perinatal epidemiology, 29(6), pp.552-561.

Aheto, J.M.K., Taylor, B.M., Keegan, T.J. and Diggle, P.J., 2017. Modelling and forecasting spatio-temporal variation in the risk of chronic malnutrition among under-five children in Ghana. Spatial and spatio-temporal epidemiology, 21, pp.37-46.

Anticona, C. and San Sebastian, M., 2014. Anemia and malnutrition in indigenous children and adolescents of the Peruvian Amazon in a context of lead exposure: a cross-sectional study. Global health action, 7(1), p.22888.

Bardosono, S., Sastroamidjojo, S. and Lukito, W., 2007. Determinants of child malnutrition during the 1999 economic crisis in selected poor areas of Indonesia. Asia Pacific journal of clinical nutrition, 16(3).

Bechir, M., Schelling, E., Hamit, M.A., Tanner, M. and Zinsstag, J., 2012. Parasitic infections, anaemia and malnutrition among rural settled and mobile pastoralist mothers and their children in Chad. Ecohealth, 9(2), pp.122-131.

Black, R.E., Victora, C.G., Walker, S.P., Bhutta, Z.A., Christian, P., De Onis, M., Ezzati, M., Grantham-McGregor, S., Katz, J., Martorell, R. and Uauy, R., 2013. Maternal and child undernutrition and overweight in low-income and middle-income countries. The lancet, 382(9890), pp.427-451.

Breslow, N.E. and Clayton, D.G., 1993. Approximate inference in generalized linear mixed models. Journal of the American statistical Association, 88(421), pp.9-25. 
El Kishawi, R.R., Soo, K.L., Abed, Y.A. and Muda, W.A.M.W., 2015. Anaemia among children aged $2-5$ years in the Gaza Strip-Palestinian: a cross sectional study. BMC Public Health, 15(1), p.319.

Gari, T., Loha, E., Deressa, W., Solomon, T., Atsbeha, H., Assegid, M., Hailu, A. and Lindtjørn, B., 2017. Anaemia among children in a drought affected community in southcentral Ethiopia. PloS one, 12(3), p. e0170898.

Gaston, R.T., Ramroop, S. and Habyarimana, F., 2018. Determinants of factors associated with anaemia among children under five years in Lesotho. African Population Studies, 32(1).

Gaston, R.T. and Ramroop, S., 2020. Prevalence of and factors associated with malaria in children under five years of age in Malawi, using malaria indicator survey data. Heliyon, 6(5), p. e03946.

Gayawan, E., Arogundade, E.D. and Adebayo, S.B., 2014. Possible determinants and spatial patterns of anaemia among young children in Nigeria: a Bayesian semi-parametric modelling. International Health, 6(1), pp.35-45.

Gueorguieva, R., 2001. A multivariate generalized linear mixed model for joint modelling of clustered outcomes in the exponential family. Statistical Modelling, 1(3), pp.177-193.

Habyarimana, F., Zewotir, T. and Ramroop, S., 2016. Joint modeling of poverty of households and malnutrition of children under five years from demographic and health survey data: case of Rwanda. Journal of Economics and Behavioral Studies, 8(2), pp.108114.

Hedeker, D., 2005. Generalized linear mixed models. Encyclopedia of statistics in behavioral science: John Wiley and Sons, Ltd.

Kassebaum, N., Kyu, H.H., Zoeckler, L., Olsen, H.E., Thomas, K., Pinho, C., Bhutta, Z.A., Dandona, L., Ferrari, A., Ghiwot, T.T. and Hay, S.I., 2017. Child and adolescent health from 
1990 to 2015: findings from the global burden of diseases, injuries, and risk factors 2015 study. JAMA Paediatrics, 171(6), pp.573-592.

Kavosi, E., Rostami, Z.H., Kavosi, Z., Nasihatkon, A., Moghadami, M. and Heidari, M., 2014. Prevalence and determinants of under-nutrition among children under six: a crosssectional survey in Fars province, Iran. International Journal of Health Policy and Management, 3(2), p.71.

Kazembe, L.N., 2013. An additive regression model for investigating the relationship between childhood health and socio-economic status. Spatial and Spatio-temporal Epidemiology, 6, pp.71-84.

Kejo, D., Petrucka, P.M., Martin, H., Kimanya, M.E. and Mosha, T.C., 2018. Prevalence and predictors of anemia among children under 5 years of age in Arusha District, Tanzania. Pediatric health, medicine and therapeutics, 9, p.9.

Kounnavong, S., Sunahara, T., Mascie-Taylor, C.N., Hashizume, M., Okumura, J., Moji, K., Boupha, B. and Yamamoto, T., 2011. Effect of daily versus weekly home fortification with multiple micronutrient powder on haemoglobin concentration of young children in a rural area, Lao People's Democratic Republic: a randomised trial. Nutrition Journal, 10(1), p.129.

Leal, L.P., Batista Filho, M., Lira, P.I.C.D., Figueiroa, J.N. and Osório, M.M., 2011. Prevalence of anaemia and associated factors in children aged 6-59 months in Pernambuco, Northeastern Brazil. Revista de Saude Publica, 45, pp.457-466.

Letuka, T. and Frade, S., 2020. Household and individual risk factors of anaemia among under-5 children in Lesotho. African Health Sciences, 20(3), pp.1478-1486.

Masibo, P.K. and Makoka, D., 2012. Trends and determinants of undernutrition among young Kenyan children: Kenya Demographic and Health Survey; 1993, 1998, 2003 and 2008-2009. Public Health Nutrition, 15(9), pp.1715-1727.

Milman, N., 2011. Anaemia-still a major health problem in many parts of the world! Annals of Hematology, 90(4), pp.369-377. 
Ministry of Health [Lesotho] and ICF International. 2016. Lesotho Demographic and Health Survey 2014. Maseru, Lesotho: Ministry of Health and ICF International.

Mohammed, S.H., Larijani, B. and Esmaillzadeh, A., 2019. Concurrent anemia and stunting in young children: prevalence, dietary and non-dietary associated factors. Nutrition journal, 18(1), pp.1-10.

Molenberghs, G. and Verbeke, G., 2005. Models for discrete longitudinal data. New York: Springer-Verlag.

Okoromah, C.A., Ekure, E.N., Lesi, F.E., Okunowo, W.O., Tijani, B.O. and Okeiyi, J.C., 2011. Prevalence, profile and predictors of malnutrition in children with congenital heart defects: a case-control observational study. Archives of Disease in Childhood, 96(4), pp.354360.

Oliveira, D., Ferreira, F.S., Atouguia, J., Fortes, F., Guerra, A. and Centeno-Lima, S., 2015. Infection by intestinal parasites, stunting and anemia in school-aged children from southern Angola. PloS one, 10(9), p. e0137327.

Olney, D.K., Kariger, P.K., Stoltzfus, R.J., Khalfan, S.S., Ali, N.S., Tielsch, J.M., Sazawal, S., Black, R., Allen, L.H. and Pollitt, E., 2009. Development of nutritionally at-risk young children is predicted by malaria, anaemia, and stunting in Pemba, Zanzibar. The Journal of Nutrition, 139(4), pp.763-772.

Rahman, M.S., Mushfiquee, M., Masud, M.S. and Howlader, T., 2019. Association between malnutrition and anaemia in under-five children and women of reproductive age: Evidence from Bangladesh Demographic and Health Survey 2011. PloS one, 14(7), p. e0219170.

Renzaho, A.M.N., 2006. Mortality, malnutrition and the humanitarian response to the food crises in Lesotho. Journal of Emergency Primary Health Care, 4(4).

Rivadeneira, M.F., Moncayo, A.L., Tello, B., Torres, A.L., Buitrón, G.J., Astudillo, F., Fredricks, T.R. and Grijalva, M.J., 2020. A Multi-Causal Model for Chronic Malnutrition and 
Anaemia in a Population of Rural Coastal Children in Ecuador. Maternal and Child Health Journal, 24(4), pp.472-482.

Sachdev, H.P.S., Gera, T. and Nestel, P., 2005. Effect of iron supplementation on mental and motor development in children: systematic review of randomised controlled trials. Public Health Nutrition, 8(2), pp.117-132.

Setboonsarng, S., 2005. Child malnutrition as a poverty indicator: an evaluation in the context of different development interventions in Indonesia (No. 21). ADB Institute Discussion Papers.

Tuerlinckx, F., Rijmen, F., Verbeke, G. and De Boeck, P., 2006. Statistical inference in generalized linear mixed models: A review. British Journal of Mathematical and Statistical Psychology, 59(2), pp.225-255.

UNICEF. (2017). State of the World's children: Children in a digital World. New York, NY: UNICEF.

Verbeke, G. and Molenberghs, G., 2003. The use of score tests for inference on variance components. Biometrics, 59(2), pp.254-262.

Worku, Z., 2003. Malnutrition among rural and urban children in Lesotho: related hazard and survival probabilities. Health SA Gesondheid, 8(3), pp.98-109.

World Health Organization, 2011. Haemoglobin concentrations for the diagnosis of anaemia and assessment of severity. Geneva: World Health Organization, 2011.

Yang, W., Li, X., Li, Y., Zhang, S., Liu, L., Wang, X. and Li, W., 2012. Anemia, malnutrition and their correlations with socio-demographic characteristics and feeding practices among infants aged 0-18 months in rural areas of Shaanxi province in north western China: a cross-sectional study. BMC Public Health, 12(1), pp.1-7. 
